\title{
Comparison of the Diagnostic Value of Mammography and Ultrasonography for Breast Ductal Carcinoma in situ
}

\author{
WEN XIAN CHEN, JI SHEN AND XIAO LIU* \\ Department of Ultrasonic, Huzhou Central Hospital, No. 198 Hongqi Road, Huzhou, Zhejiang 313000, China
}

Chen et al.: Mammography and ultrasonography in breast ductal carcinoma

\begin{abstract}
To evaluate the diagnostic value and imaging features of mammography and contrast-enhanced ultrasonography in breast ductal carcinoma in situ, 60 patients with 62 lesions were selected from 80 patients with ductal carcinoma in situ who were surgically treated and pathologically diagnosed in Huzhou Central Hospital from June 2017 to June 2019. All patients were examined by contrast-enhanced ultrasonography and mammography within $2 \mathrm{w}$ before the operation. The imaging features, diagnostic accuracy, sensitivity, specificity, positive predictive value and negative predictive value of the two methods were analysed. Compared to mammography, sensitivity, specificity, accuracy, positive predictive value and negative predictive value of the contrast-enhanced ultrasound in the diagnosis of benign and malignant breast ductal lesions was significantly better. In terms of imaging features, the contrast-enhanced ultrasound was significantly better than mammography in detecting the boundary and edge of lesions and calcification of lesions, but there was no significant difference between the two methods in the detection of size of the lesions. Contrast-enhanced ultrasound could provide a rapid, reliable, and accurate diagnostic basis for the diagnosis of ductal carcinoma in situ and it is of great significance for the diagnosis and treatment of ductal carcinoma in situ in the clinic. Mammography and contrast-enhanced ultrasound can be combined to improve the accuracy and sensitivity of early diagnosis and screening of ductal carcinoma in situ, which is worthy of clinical application.
\end{abstract}

Key words: Ductal carcinoma in situ, contrast-enhanced ultrasonography, mammography

The incidence of ductal carcinoma in situ (DCIS) was high in the breast diseases and its common clinical symptoms were mainly characterized by the breast mass, nipple discharge, and pain ${ }^{[1]}$. Studies have found that about $50 \%$ of the breast DCIS patients were associated with nipple discharge and accompanied by pain from breast inflammation and ductal ectasia ${ }^{[2]}$. At present, the common clinical screening and examination methods mainly included mammography, MRI ductography, and contrast-enhanced ultrasonography examination. In recent years, the advantages of the contrast-enhanced ultrasonography in the early screening and diagnosis of the breast DCIS have become increasingly prominent, mainly because the ultrasound has the advantages of convenient, noninvasive, no side effects, economic, real-time dynamic. Contrast-enhanced ultrasound was a dynamic and realtime imaging technology, which can display the spatial distribution and blood perfusion difference of micro vessels around and inside the mass dynamically, and provide a reliable basis for the doctors to diagnose the benign and malignant tumours. However, there were few studies on the contrast-enhanced ultrasound in the breast intraductal lesions, so this study mainly discusses the application value of contrast-enhanced ultrasound in the diagnosis of breast intraductal lesions.Clinical data of 80 patients with breast intraductal lesions diagnosed by pathology in Huzhou Central Hospital from June 2017 to June 2019 was collected. Inclusion criteria were, unilateral, only DCIS, no invasive components, no previous breast cancer or other malignant tumours, received mammography and bilateral breast ultrasound evaluation, no previous chemotherapy or radiotherapy, no pregnancy or lactation. A total of 60 patients with 62 lesions were included in this study, with an age of $55.71 \pm 8.94 \mathrm{y}$. All patients were examined by the contrast-enhanced ultrasound and mammography within $2 \mathrm{w}$ before the operation.GE Logiq E9 color Doppler ultrasound diagnostic instrument (USA) was used in the contrastenhanced ultrasound, which has a real-time contrastenhanced function. According to the breast detection 
parameters preset by the default instrument, the patient uses the dorsal decubitus to facilitate a comprehensive examination of both sides of the breast. The doctor adjusts the appropriate parameters according to the patient's lesion condition to obtain the best gray-scale ultrasound and colour blood flow images. The pathological features of the lesions were recorded, including shape, size, boundary, internal echo and internal calcification. The blood supply of the lesions was evaluated according to the Adler semi-quantitative method. Mammography was performed with Selenia dimensional mammography system. Patients were routinely examined in bilateral mammary lateral oblique position (MLO position) and axial position (CC position). The shape, size, margin, and calcification of the breast were observed. The contrast-enhanced ultrasound images and breast mammography images were interpreted and confirmed by two experienced clinical imaging doctors. The size, shape, location, lesion boundary, and imaging features of the two methods were compared. The diagnostic accuracy, sensitivity, specificity, positive predictive value and negative predictive value of the two methods were compared.SPSS 18.0 software was used for the statistical analysis. The qualitative data were expressed as percentage and the quantitative data were expressed as mean $\pm \mathrm{SD}$. An independent sample t-test was used to

\section{TABLE 1: THE NUMBER AND MORPHOLOGY OF LESIONS DETECTED BY THE TWO METHODS \\ Contrast-

enhanced
ultrasound $X$-ray group $\mathrm{X}^{2} \quad \mathrm{p}$ value
group

$\begin{array}{lllll}\begin{array}{l}\text { Number of } \\ \text { lesions }\end{array} & & & 10.263 & 0.037 \\ \text { Detected } & 42 & 32 & & \\ \text { Not detected } & 20 & 30 & & \\ \text { Tumour sharp } & & & 16.731 & 0.013 \\ \text { Oval } & 44 & 29 & & \\ \text { Irregular } & 18 & 33 & & \end{array}$

compare the quantitative data accord with a normal distribution, and Mann-Whitney U test was used for the quantitative data that did not accord with a normal distribution. $p<0.05$ means the difference was statistically significant. The number and morphology of lesions detected by the 2 methods were shown in Table 1. The results showed that there were significant differences in the number and morphology of lesions detected by the contrast-enhanced ultrasound group, and the difference was statistically significant $(p<0.05)$. Two detection methods were used to detect the edge and boundary of the lesion, and the results were shown in fig. 1 . The results showed that the difference between the two methods in detecting the boundary and edge of lesions was obvious. The detection result of the contrast-enhanced ultrasound group was better than that of the mammography group $(p<0.05)$.By analysing the detection of calcification of the two methods (Table 2), it was found that the contrast-enhanced ultrasound was more suitable for the detection of calcification. There was a significant difference between the two groups $(p<0.05)$. Contrastenhanced ultrasound and mammography were used to compare the sensitivity, specificity, accuracy, positive predictive value, and negative predictive value of the benign and malignant breast intraductal lesions (Table 3). There was a significant difference in the diagnostic efficiency between the two methods $(p<0.05)$. The results showed that the diagnostic ability of contrast-enhanced ultrasound was better than that of mammography in the differential diagnosis of benign and malignant breast intraductal lesions. The ability of the two methods to detect the accuracy of lesion size was analysed. The results showed that the average preoperative/postoperative size deviation of the contrast-enhanced ultrasound was $14.6 \mathrm{~mm}$, and the mammography was $12.4 \mathrm{~mm}$ (underestimated by both methods). The mean square error (MSE) analysis showed that when the tumour size was more than $2 \mathrm{~cm}$, the detection accuracy decreased gradually (Table 4).
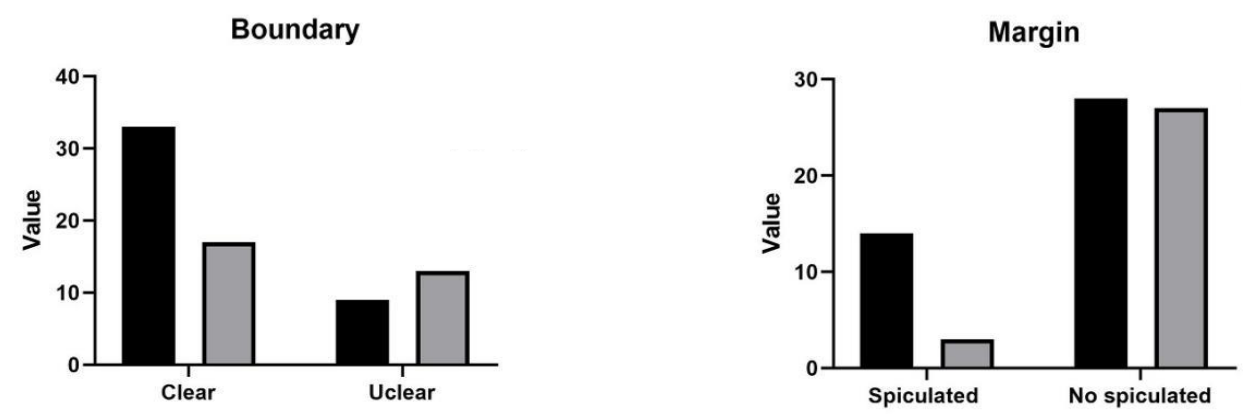

Fig. 1: Two detection methods to detect the edge and boundary of the lesions 
TABLE 2: THE DETECTION OF CALCIFICATION OF THE TWO METHODS

\begin{tabular}{|c|c|c|c|c|}
\hline & Contrast-enhanced ultrasound group & Mammography group & $\times 2$ & $\mathrm{p}$ value \\
\hline Calcifications & & & 19.909 & 0.005 \\
\hline Calcifications & 29 & 45 & & \\
\hline No Calcifications & 33 & 17 & & \\
\hline
\end{tabular}

TABLE 3: COMPARISON OF THE EFFICACY OF THE TWO DETECTION METHODS IN THE DIAGNOSIS OF BENIGN AND MALIGNANT BREAST INTRADUCTAL LESIONS

\begin{tabular}{lccccc}
\hline Groups & Sensitivity/\% & Specificity/\% & Accuracy/\% & $\begin{array}{c}\text { Positive predictive } \\
\text { value/\% }\end{array}$ & $\begin{array}{c}\text { Negative predictive } \\
\text { value/\% }\end{array}$ \\
\hline Mammography & 31.03 & 50.31 & 66.28 & 69.21 & 65.50 \\
Contrast-enhanced & 75.86 & 66.40 & 72.42 & 64.57 & 81.10 \\
ultrasound & 0.001 & 0.012 & 0.053 & 0.061 & 0.019 \\
p value & & &
\end{tabular}

There was no significant difference in the size of lesions between the two groups $(\mathrm{p}<0.05)$.DCIS was a kind of tumour with intraductal lesions, which grows only in the basal intima and has the possibility of developing into invasive malignant breast cancer. It was a common sign of early breast cancer. The early DCIS lesions are relatively small, so it is difficult to diagnose accurately with the common clinical methods. The contrastenhanced ultrasound and mammography can detect lesions or calcifications smaller than $1 \mathrm{~cm}$, but the detection rate of mammography is only $16 \%{ }^{[3]}$. By comparing and analysing the difference of image features between the mammography and contrastenhanced ultrasound, this paper discusses the application value of these two methods in the preoperative diagnosis of DCIS. Early diagnosis of breast disease contributes to early treatment and better prognosis. The contrast-enhanced ultrasound was a good method for the early diagnosis of breast diseases, which was helpful for the classification of benign and malignant breast masses. In this study, the overall sensitivity, specificity, and accuracy of the contrastenhanced ultrasound in the diagnosis of breast lesions was $75.86,66.4$ and $72.42 \%$, respectively. The sensitivity was similar to that in the contrast-enhanced ultrasound meta-analysis report (76\%), but the specificity was lower than that in the meta-analysis (79\%). In this study, the contrast-enhanced ultrasound was superior to mammography in sensitivity $(31.03 \%$ sensitivity and $50.31 \%$ specificity), which may be related to lesion calcification and pathological structure, such as micro vessel density (MVD) and interstitial structure. Therefore, the contrast-enhanced ultrasound is a good method for the early screening of DCIS. Clinically, the contrast-enhanced ultrasound was a dynamic and real-time imaging technology and its contrast agent was non-toxic to humans, so it has the advantages of economy, convenience and no radiation, which was the main means for the early diagnosis of breast cancer ${ }^{[4,5]}$. In this study, the proportion of the irregular shape, unclear boundary, burr and uneven edge detected by the contrast-enhanced ultrasound was higher than that of mammography $(\mathrm{p}<0.05)$, which was similar to the results of Tang et al. ${ }^{[6]}$. The pathological changes corresponding to the above ultrasonic signs were related to the infiltration of the lesion parenchyma to the surrounding tissue, accompanied by different degrees of interstitial reaction, forming an irregular boundary. Mammography was easily covered or overlapped by the dense breast glands, or affected by the location of lesions ${ }^{[7,8]}$. The contrast-enhanced ultrasound can display the lesions and their marginal features clearly, which is helpful to make a qualitative diagnosis. Early breast cancer lesions were prone to calcification, which often lead to the breast intraductallesions ${ }^{[9]}$. Current studies suggested that the calcification mainly occurs in the atypical hyperplasia stage of the breast, because the breast hyperplasia can lead to the micro vascular hyperplasia and mass dystrophy easily. The calcification forms of the breast masses were complex and diverse, but they were mainly distributed in linear or community aggregation ${ }^{[10-12]}$. In this study, mammography and contrast-enhanced ultrasound were used to detect the breast calcification. It was found that the mammography was easier to diagnose the micro calcification in breast glands $(p<0.05)$. This may be related to the detection principle of the contrast-enhanced ultrasound and the distribution of calcification of breast glands. At the same time, ultrasound detection of the spotted calcified masses was easily disturbed by the strong echoes of the catheter wall. It was worth noting that the mammography cannot detect all patients, some patients with small calcification cannot be detected by mammography, and it was 
www.ijpsonline.com

TABLE 4: RESULTS OF TUMOR SIZE DEVIATION BETWEEN TWO GROUPS OF IMAGING METHODS

\begin{tabular}{|c|c|c|c|c|c|c|}
\hline & & Patho & gy minus ultrasound & Patho & ogy minus ultrasound & $p$ value \\
\hline & $\mathrm{n}$ & Mean $(\mathrm{mm})$ & Level of agreement $(\mathrm{mm})$ & Mean $(\mathrm{mm})$ & Level of agreement $(\mathrm{mm})$ & \\
\hline overall & 62 & 14.6 & $-75 \sim 46$ & 12.4 & $-48 \sim 54$ & 0.27 \\
\hline Size $\leq 2 \mathrm{~cm}$ & 36 & -3 & $-32 \sim 31$ & -6.2 & $-32 \sim 20$ & 0.12 \\
\hline Size $>2 \mathrm{~cm}$ & 26 & 32 & $-33 \sim 98$ & 30 & $-54 \sim 77$ & 0.41 \\
\hline$M S E \leq 2 \mathrm{~cm}$ & 33 & 23 & - & 20 & - & 0.20 \\
\hline$M S E>2 \mathrm{~cm}$ & 29 & 110 & - & 220 & - & 0.32 \\
\hline MSE overall & 62 & 64 & - & 110 & - & 0.16 \\
\hline
\end{tabular}

difficult to confirm by palpation. However, thy can be diagnosed by obtaining the echo changes of the glands around the lesions by contrast-enhanced ultrasound. Therefore, the contrast-enhanced ultrasound has a good diagnostic advantage in detecting breast intraductal lesions and their characteristics, which is helpful for the diagnosis, treatment, and judgment of clinicians.In summary, the conventional mammography was not adequate enough to provide reliable basis for the diagnosis of breast intraductal lesions, while the contrast-enhanced ultrasound can provide a rapid, reliable, and accurate basis for the diagnosis of breast intraductal lesions, which is of great significance in clinical diagnosis and treatment of breast intraductal lesions. The combined application of mammography and contrast-enhanced ultrasound can improve the accuracy and sensitivity of early diagnosis and screening of breast cancer, which is worth popularizing and applying in the clinic.

\section{Authors contributions:}

Wenxian Chen conceived and designed the experiments gave an experimental guidance in the lab; JiShen performed the experiments; Xiao Liu analyzed the data and wrote the paper.

\section{Acknowledgements:}

This work was supported by Huzhou Central Hospital.

\section{Conflict of interest:}

All authors report no conflicts of interest in this work.

\section{REFERENCES}

1. Hu Z, Cheng X, Li J, Jiang J, Jiang Z, Li H, et al. Preliminary study of real-time three-dimensional contrast-enhanced ultrasound of sentinel lymph nodes in breast cancer. EurRadiol 2020;30:44-53.

2. Chen F, Liu J, Zhang X, Liao H. Probability analysis of axillary lymph node metastasis in breast cancer patients using particle space-time distribution model. HealthcTechnolLett 2019;6:352-60.

3. Shimazu K, Miyake T, Tanei T, Naoi Y, Shimoda M, Kagara N, et al. Real-Time Visualization of Lymphatic Flow to Sentinel Lymph Nodes by Contrast-Enhanced Ultrasonography with Sonazoid in Patients with Breast Cancer. Ultrasound Med Biol 2019;45:2634-40.

4. Kinoshita S, Miyake R, Shimada N, Hirano A, Seki Y, Shimizu $\mathrm{K}$, et al. Initial experience with contrast-enhanced ultrasonography in follow-up assessment of small breast cancer treated by cryoablation. Aust Med J 2017;10:12-9.

5. Shimazu K, Miyake T, Tanei T, Naoi Y, Shimoda M, Kagara N, et al. Real-Time Visualization of Lymphatic Flow to Sentinel Lymph Nodes by Contrast-Enhanced Ultrasonography with Sonazoid in Patients with Breast Cancer. Ultrasound Med Biol 2019;12:54-67.

6. Li JT, Zhao HM, Guo XH, Tian PQ, Lü MH, Li LF, et al. Preoperative evaluation of sentinel lymph node biopsy using contrast-enhanced ultrasonography in early breast cancer patients and the involved disturbing factors. Zhonghua Yi XueZaZhi 2019;99:1086-9.

7. Wang M, Feng HL, Liu YQ, Liu H, Jiang YX, Zhu QL, et al. Angiogenesis Research in Mouse Mammary Cancer Based on Contrast-enhanced Ultrasonography. AcadRadiol 2018;31:107-118.

8. Nakamura K, Ohira M, Noda S. Investigation of four cases of mucinous breast carcinoma that underwent contrast-enhanced ultrasonography with Sonazoid. ChoonpaIgaku 2019;21:112-7.

9. Kazutaka N. Expectations for contrast-enhanced ultrasonography. J Medi Ultrasonic 2019;46:285-6.

10. Banzato T, Burti S, Rubini G, Orlandi R, Bargellini P, Bonsembiante $\mathrm{F}$, et al. Contrast-enhanced ultrasonography features of hepatobiliary neoplasms in cats. Vet Rec 2020;186:320.

11. Streb JW, Tchelepi H, Malhi H, Deurdulian C, Grant EG. Retrospective Analysis of Contrast-enhanced Ultrasonography Effectiveness in Reducing Time to Diagnosis and Imagingrelated Expenditures at a Single Large United States County Hospital. Ultrasound Q 2019;35:95-102.

12. Lee YJ, Kim SH, Kang BJ, Kim YJ. Contrast-Enhanced Ultrasound for Early Prediction of Response of Breast Cancer to Neoadjuvant Chemotherapy. Eur J Ultrasound 2019;40:194204.

This is an open access article distributed under the terms of the Creative Commons Attribution-NonCommercial-ShareAlike 3.0 License, which allows others to remix, tweak, and build upon the work non-commercially, as long as the author is credited and the new creations are licensed under the identical terms

This article was originally published in a special issue, "Biomedical Research in Healthcare Setting" Indian J Pharm Sci 2020:82(3)Spl issue5;115-118 